\title{
Tratamiento del asma severo con Omalizumab: experiencia pediátrica en Chile y revisión de la literatura
}

\author{
ANA MARÍA HERRERA G.* JURY HERNÁNDEZ C.**, JOSÉ ANTONIO PERILLÁN T.***, \\ VIVIANA LEZANA S.****, CECILIA ÁLVAREZ G.*****, MARÍA ANGÉLICA MARINOVIC M.******, \\ PAMELA NAVARRETE C.**, CAROLINA CRUZ B.******** y LUIS CAMPUSANO T.********
}

\author{
Omalizumab treatment on severe asthma patients: pediatric experience in Chile and literature \\ review
}

This paper presents clinical experience with Omalizumab treatment in 8 pediatric patients in Chile. All children presented difficult to control asthma despite receiving high intensity treatment, with low quality of life. All patients were studied in order to discard errors in asthma diagnosis and to evaluate asthma treatment adherence and inhalation technique. After evaluation, patients proven to have severe therapy resistant asthma were indicated treatment with Omalizumab. Significant clinical improvement was observed, with reduced asthma symptoms and number of exacerbations, as well as an improved quality of life. Omalizumab showed a good safety profile with mild and transient adverse reactions in 6 administrations of a total of 122. of life.

Key words: Omalizumab; asthma; anti-asthmatic agents; antibodies, anti-idiotypic; child; quality

\section{Resumen}

Se presenta la experiencia clínica con el uso de Omalizumab en 8 pacientes pediátricos en nuestro país. Todos los pacientes presentaban asma sin control a pesar de recibir terapia de alta intensidad, asociado a una muy deficiente calidad de vida. La totalidad de los pacientes fueron sometidos en cada centro a un estudio exhaustivo para poder descartar error en el diagnóstico y se evaluó la adherencia y la técnica inhalada. Al comprobarse que estos pacientes tenían asma severo resistente a tratamiento se indicó Omalizumab, el cual produjo una mejoría clínica significativa. Se observó una reducción de las exacerbaciones y de los sintomas de asma acompañado de una mejoría de la calidad de vida, asociado a un buen perfil de seguridad. Se observaron reacciones adversas leves y transitorias en 6 administraciones de un total de 122.

Palabras clave: Omalizumab; asma; agentes antiasmáticos; anticuerpos; anti-idiotípicos; niños; calidad de vida.

\section{Introducción}

La gran mayoría de los niños con asma logra un control adecuado de la enfermedad con dosis bajas y seguras de corticoides inhalados. Sin embargo, existe un grupo de pacientes cercano al $5 \%$ que persiste con un mal control pese al uso de terapia máxima. Para este grupo de pacientes las guías internacionales para el manejo del asma bronquial proponen el uso de terapias biológicas

\footnotetext{
* Clínica Santa María y Universidad de los Andes. Santiago.

** Complejo Asistencial Dr. Víctor Ríos Ruiz. Los Ángeles, Región del Bío-Bío.

*** Hospital San Juan de Dios y Universidad de Chile. Santiago.

**** Universidad de Valparaíso.

***** Clínica Alemana de Santiago y Universidad del Desarrollo.

****** Clínica Santa María. Santiago.

******* Hospital Roberto del Río. Santiago.

$* * * * * * * *$ Universidad de Antofagasta.
} 
como Omalizumab. Este es un anticuerpo monoclonal recombinante humano anti Inmunoglobulina $\mathrm{E}$ (IgE) que se une a la porción Fc de la $\operatorname{IgE}$ libre inhibiendo la unión de ésta a los receptores de alta afinidad involucrados en la activación celular y la liberación de mediadores inflamatorios. Omalizumab fue aprobado por la Food and Drug Administration (FDA) hace más de una década y en Chile el Instituto de Salud Pública lo aprobó el año 2009 para pacientes pediátricos a partir de los 6 años. En nuestro país 8 niños con asma severo han sido tratados con este medicamento. El presente artículo tiene como objetivo mostrar la experiencia con Omalizumab en niños y hacer una revisión bibliográfica del tema.

\section{Casos clínicos}

En Chile 8 pacientes pediátricos con asma han sido tratados con Omalizumab, 5 de género masculino y 3 de género femenino, con un promedio de edad de 12 años (rango 7 a 15) (Tabla 1). Del total de pacientes 4 residen en Santiago, 2 en Los Ángeles, 1 en Viña del Mar y el último en Antofagasta. La mediana de ACT (Asthma Control Test) previo al inicio de la terapia con Omalizumab fue de 9 (rango 2 a 23). Todos los pacientes reportaban consultas al Servicio de Urgencia por crisis de asma en promedio 6 veces por año (rango 1 a 12), con curas de corticoides sistémicos en promedio 6 por año (rango 2 a 12) y con 2 hospitalizaciones en promedio por año (rango $0 \mathrm{a}$ 4). Sólo un paciente no registra hospitalizaciones previas al uso de Omalizumab y en 5 pacientes al menos una de las hospitalizaciones fue en Unidad de Paciente Crítico. Además de las exacerbaciones la mayoría de los pacientes presentaban tos persistente y disnea tanto de día como de noche, con despertares nocturnos, uso de salbutamol e incapacidad para realizar actividad física. Sólo dos pacientes eran poco sintomáticos entre las crisis. Todos los pacientes estaban sensibilizados a alérgenos perennes. En promedio los pacientes presentaron sensibilización a 3 alérgenos en el test cutáneo (rango 1 a 6), siendo el más frecuentemente pesquisado el polvo de habitación seguido por pastos, malezas y caspa de gato. A pesar de que todos los pacientes eran alérgicos los niveles de $\operatorname{IgE}$ fueron bastante variables con un rango entre 79 y $9300 \mathrm{UI} / \mathrm{L}$. Con respecto a la función pulmonar, la mitad de los pacientes tenían niveles de $\mathrm{VEF}_{1}$ bajo $80 \%$ del predicho, en la otra mitad la función pulmonar era normal. En 7 de los 8 pacientes se registraron comorbilidades siendo la más frecuente la rinitis alérgica presente en 7 pacientes, seguida por dermatitis atópica. Todos los pacientes de la serie tienen estudio tendiente a descartar error en el diagnóstico de asma. Entre los exámenes destaca el test del sudor y recuento de inmunoglobulinas A, M, G y E, realizados en la totalidad de los niños, en 5 se efectuó además tomografía axial computarizada (TAC) de tórax, en 2 determinación anticuerpos antineumococo y en otros 2 estudio de microscopía electrónica para descartar disquinesia ciliar. Otros exámenes realizados a algunos de los pacientes fueron serología para Toxocara, ecocardiografía, determinación de alfa 1 antitripsina, niveles de vitamina $\mathrm{D}$, subpoblaciones de linfocitos, subclases de IgG, TAC de cavidades perinasales y fibrobroncoscopía. Con respecto al tratamiento 2 pacientes estaban recibiendo terapia con dos controladores (corticoides inhalados más beta 2 agonistas de acción prolongada, LABA), tres pacientes recibían tres controladores (corticoides inhalados, LABA y Montelukast en 2 casos y corticoides orales en otro), dos recibían cuatro controladores (corticoides inhalados, LABA, Montelukast y Azitomicina) y el último estaba siendo tratado concomitantemente con 5 medicamentos controladores (corticoides inhalados, LABA, teofilina retard, Montelukast y Azitromicina). El segundo controlador más frecuentemente utilizado fue LABA seguido por Montelukast y Azitromicina. Sólo un paciente estaba en tratamiento con corticoides orales en forma permanente. Todos los pacientes tenían un muy mal control de su enfermedad pese a estar recibiendo terapia de alta intensidad. Además, tenían una muy mala calidad de vida debido a las múltiples exacerbaciones que presentaban, lo que motivaba consultas repetidas a los Servicios de Urgencia, hospitalizaciones, ausentismo escolar y limitación de la capacidad de ejercicio y de las actividades de la vida diaria por la presencia de síntomas persistentes. En cada centro estos pacientes fueron estudiados en forma exhaustiva. En todos los casos se confirmó el diagnóstico de asma mediante una detallada historia clínica y examen físico y mediante el uso de espirometría y pruebas de provocación bronquial. Mediante distintos exámenes se descartaron otras patologías que pudieran confundirse con asma bronquial y la presencia de comorbilidades que pudieran incidir negativamente en la evolución del asma. Se evaluó clínicamente la adherencia al tratamiento, la técnica inhalada y los factores ambientales intradomiciliarios como exposición a alérgenos y a contaminantes como humo de cigarrillo y estufas a leña o parafina. Luego de esta evaluación, al no encontrarse causas que explicaran el mal control y por tratarse de pacientes con asma alérgico en etapa 4 de GINA, se decidió 


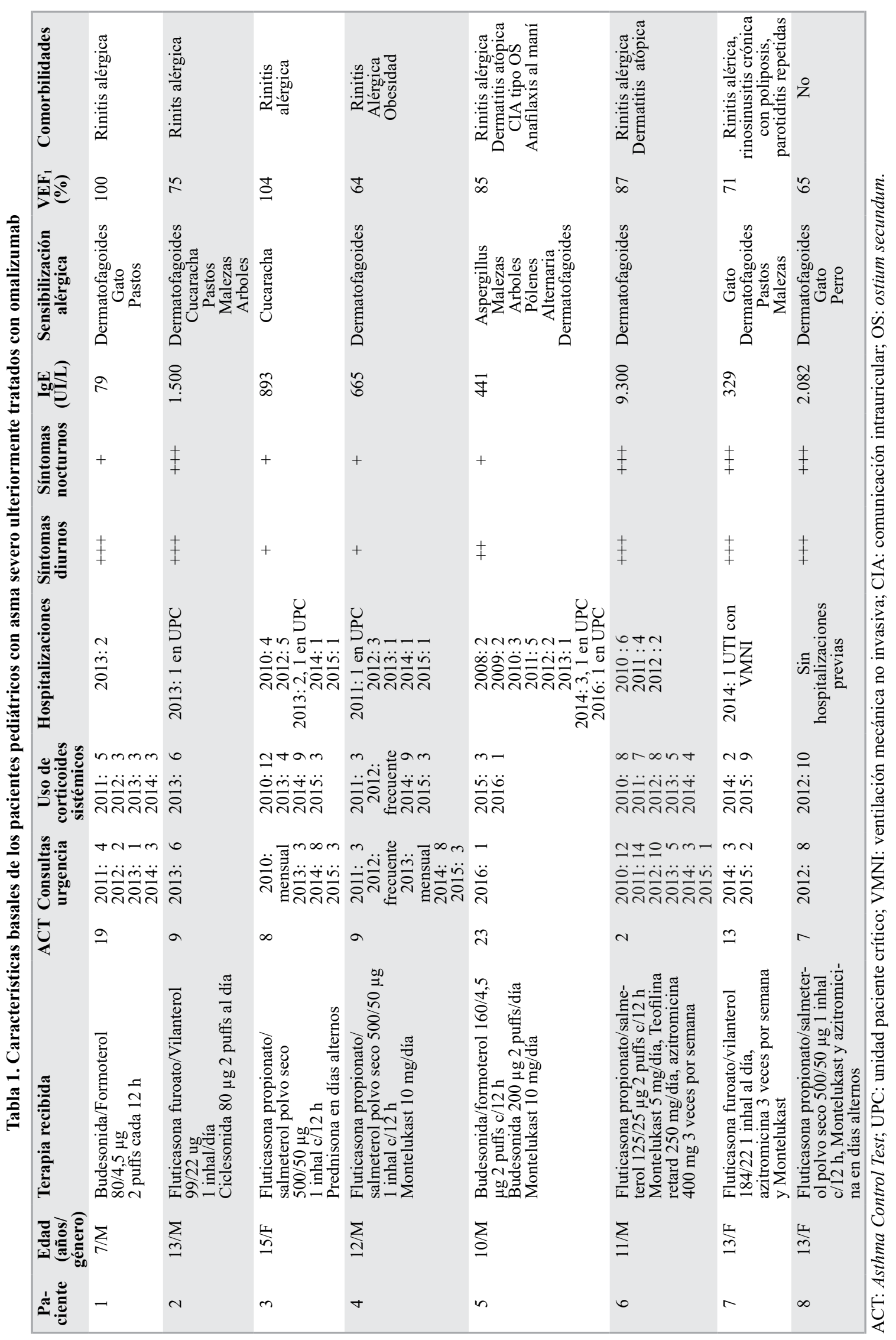


escalar en la terapia a una etapa 5, utilizando Omalizumab. Todos los pacientes tratados con este medicamento experimentaron una mejoría del control de su asma bronquial, incluso antes de los 4 meses de iniciado su uso (Tabla 2). Esta mejoría es objetivada por disminución en el número de consultas al Servicio de Urgencia, curas con corticoides orales, hospitalizaciones y mejoría de los síntomas medido por el cuestionario Asthma Control Test (ACT). Por otro lado, la totalidad de los pacientes con síntomas importantes entre las crisis experimentaron una disminución de éstos, lo que les permitió volver a realizar actividad física, con una mejoría significativa de su calidad de vida. De un total de 122 dosis de omalizumab administradas, sólo en 6 se observaron efectos adversos, los cuales fueron leves: erupción cutánea transitoria en 2 pacientes, cefalea en 2 pacientes, fiebre en un paciente y náuseas en otro. El tiempo promedio de tratamiento en nuestra serie fue de 12 meses (rango 4 a 22). En 4 pacientes la terapia es mantenida hasta el día de hoy, en 3 pacientes esta se suspendió por problemas de costo y en uno por buena evolución luego de 1 año de tratamiento.

\section{Fisiopatología del asma alérgico}

El asma bronquial se caracteriza por la presencia de una inflamación crónica de la vía aérea. En

Tabla 2. Evolución de los pacientes luego del uso de omalizumab

\begin{tabular}{|c|c|c|c|}
\hline Paciente & Como se objetiva mejoría & $\begin{array}{l}\text { Tiempo de } \\
\text { tratamiento }\end{array}$ & $\begin{array}{l}\text { Reacciones } \\
\text { adversas }\end{array}$ \\
\hline 1 & $\begin{array}{l}\text { ACT } 25 \\
\text { Desaparecen síntomas diurnos y nocturnos } \\
\text { Sólo una cura corticoidal } \\
\text { Sin hospitalizaciones } \\
\text { Sin consultas a Servicio de Urgencia } \\
\text { Sin ausentismo escolar } \\
\text { Realizando educación física } \\
\text { Mejoría calidad de vida }\end{array}$ & 1 año & $\begin{array}{l}\text { Fiebre } \\
\text { Erupción cutánea pasajera }\end{array}$ \\
\hline 2 & $\begin{array}{l}\text { ACT } 16 \\
\text { Disminución significativa síntomas diurnos y nocturnos } \\
\text { Sin uso de corticoides } \\
\text { Sin hospitalizaciones } \\
\text { Sin consultas a Servicio de Urgencia }\end{array}$ & 15 meses & Ninguna \\
\hline 3 & $\begin{array}{l}\text { ACT } 23 \\
\text { Sin uso corticoides } \\
\text { Sin hospitalizaciones } \\
\text { Consulta Servicio de Urgencia sólo una vez }\end{array}$ & 6 meses & Ninguna \\
\hline 4 & $\begin{array}{l}\text { ACT } 25 \\
\text { Sólo una cura corticoidal } \\
\text { Sin hospitalizaciones } \\
\text { Sin consultas a Servicio de Urgencia }\end{array}$ & $\begin{array}{l}\text { 2013: } 6 \text { meses } \\
\text { reinicia en mayo } \\
\text { 2015: } 16 \text { meses }\end{array}$ & $\begin{array}{l}\text { Eritema transitorio en sitio } \\
\text { de inyección }\end{array}$ \\
\hline 5 & $\begin{array}{l}\text { ACT } 23 \\
\text { Sin síntomas diurnos ni nocturnos } \\
\text { Sin consultas a Servicio de Urgencia } \\
\text { Sin hospitalizaciones } \\
\text { Se reintegra a equipo basketball }\end{array}$ & 5 meses & Ninguna \\
\hline 6 & $\begin{array}{l}\text { ACT } 15 \\
\text { Sin uso de corticoides } \\
\text { Sin hospitalizaciones }\end{array}$ & 17 meses & Cefalea \\
\hline 7 & $\begin{array}{l}\text { ACT } 23 \\
\text { Sin consultas a Servicio de Urgencia } \\
\text { Sin hospitalizaciones } \\
\text { Sin uso de corticoides } \\
\text { Sin ausentismo escolar } \\
\text { Realizando educación física }\end{array}$ & 12 meses & Ninguna \\
\hline 8 & $\begin{array}{l}\text { ACT } 22 \\
\text { Sólo una consulta a Servicio de Urgencia } \\
\text { Mejoría de la calidad de vida }\end{array}$ & 4 meses & $\begin{array}{l}\text { Prurito local leve, cefalea } \\
\text { leve y náuseas en una } \\
\text { ocasión }\end{array}$ \\
\hline
\end{tabular}

ACT: Asthma Control Test. 
el adulto se han descrito distintos fenotipos de asma bronquial, asociados a distintos patrones inflamatorios ${ }^{1}$. En los niños, a diferencia de lo que ocurre en los adultos, el principal mecanismo inflamatorio es de tipo alérgico, pudiendo presentarse con distinto grado de severidad y compromiso de la función pulmonar ${ }^{2,3}$. En nuestra serie observamos que la totalidad de los niños eran portadores de asma alérgico dado por la presencia de test cutáneo positivo, $\operatorname{IgE}$ alta y comorbilidades como rinitis alérgica y dermatitis atópica. Los pacientes alérgicos expresan IgE específica para los alérgenos a los cuales están sensibilizados y ésta se va a fijar a los receptores de alta afinidad presentes en la superficie de mastocitos, basófilos y células dendríticas ${ }^{3}$. Cuando dos moléculas de IgE toman contacto con el alérgeno se produce la degranulación del mastocito, con lo que se libera histamina, leucotrienos y prostaglandinas, causando una reacción de hipersensibilidad inmediata tipo I. Esta reacción se caracteriza por contracción del músculo liso bronquial, aumento de la secreción de mucus por parte de las glándulas submucosas y de las células caliciformes y edema de la pared bronquial por aumento de la permeabilidad vascular. Todo lo anterior causa la broncoconstricción característica de la exacerbación de asma ${ }^{4}$. Los mastocitos también liberan citoquinas que van a atraer a la mucosa respiratoria eosinófilos y linfocitos. La llegada de eosinófilos se ve potenciada por la liberación de IL-5 por parte de los linfocitos $\mathrm{TH} 2^{5}$. Los eosinófilos son las células más importantes en la respuesta alérgica tardía y son las responsables de la mantención de la inflamación en la mucosa respiratoria al liberar sustancias proinflamatorias como proteínas básicas, leucotrienos y citoquinas.

\section{Mecanismos de acción de omalizumab}

Omalizumab es un anticuerpo monoclonal humanizado anti IgE aprobado por la FDA el año 2003 y por el Instituto de Salud Pública de nuestro país el 2009 para niños mayores de 6 años. Omalizumab actúa bloqueando la IgE libre circulante, evitando que ésta se una a su receptor de alta afinidad ubicado en la superficie de mastocitos, basófilos y células dendríticas, reduciendo así la respuesta alérgica. Al haber menor cantidad de IgE libre circulante, disminuye secundariamente la expresión de receptores de alta afinidad, aumentado el efecto inhibitorio sobre la cascada alérgica $^{6,7}$. Se ha demostrado además que Omalizumab disminuye la capacidad de las células dendríticas para inducir la proliferación de linfo- citos TH2, lo que lleva a una disminución de la secreción de citoquinas como IL-4, IL-13 e IL- $5^{8}$, con una reducción del número de eosinófilos en esputo y en la mucosa bronquial ${ }^{9}$. De esta manera Omalizumab no sólo evita la degranulación del mastocito frente a la presencia del alérgeno, inhibiendo la fase temprana de la respuesta alérgica, sino que también reduce indirectamente la respuesta alérgica tardía al disminuir el reclutamiento de eosinófilos. Estudios recientes in vitro han demostrado que Omalizumab podría evitar la remodelación de la vía aérea, al disminuir la proliferación de células de músculo liso bronquial, el depósito de matriz extracelular y colágeno producto del bloqueo de la acción estimuladora de IgE sobre las células del músculo liso bronquial ${ }^{8}$. Finalmente se ha observado en estudios ex vivo que Omalizumab es capaz de mejorar la secreción de interferón alfa en monocitos de pacientes asmáticos incubados con rinovirus, mejorando de esta manera la respuesta antiviral ${ }^{9}$.

\section{Indicaciones de omalizumab en asma pediátrico}

Omalizumab está indicado en niños mayores de 6 años con asma severo resistente a tratamiento, en los cuales no se logra el control de la enfermedad a pesar de recibir terapia en etapa 4 de GINA $^{10}$. Para poder afirmar que un paciente tiene asma severo resistente a tratamiento primero se requiere de una evaluación exhaustiva, idealmente realizada por un médico especialista en enfermedades respiratorias infantiles o inmunólogo. En esta evaluación se debe confirmar el diagnóstico de asma y al mismo tiempo se debe excluir causas secundarias que pudieran confundirse con asma, tales como fibrosis quística, inmunodeficiencias y bronquiolitis obliterante entre otras. Además, se debe descartar la presencia de comorbilidades que no estén siendo adecuadamente tratadas y que pudieran incidir negativamente en la evolución del asma bronquial, como rinitis alérgica, reflujo gastroesofágico y obesidad ${ }^{11-13}$. En tercer lugar, se debe evaluar la adherencia al tratamiento que el paciente tiene indicado y la técnica inhalatoria. Este punto es fundamental ya que se estima que la principal causa de asma mal controlado es una mala adherencia al tratamiento. Finalmente se debe indagar acerca de la exposición ambiental a alérgenos a los cuales el paciente esté sensibilizado y la exposición a contaminantes intradomiciliarios como estufas a leña o parafina y el tabaquismo pasivo o activo, sobretodo en adolescentes. Sólo una vez que se 
ha cumplido a cabalidad con esta evaluación y se han descartado todos los factores que son potencialmente modificables, es posible escalar en la terapia $^{11-13}$. Para indicar Omalizumab el paciente además debe estar sensibilizado al menos a un alérgeno perenne en el test cutáneo o en la determinación de $\operatorname{IgE}$ específica y tener niveles de $\operatorname{IgE}$ total entre 30 a 1500 UI. En nuestra serie todos los pacientes fueron sometidos a una evaluación que incluyó historia y examen físico detallado y una batería de exámenes para excluir error en el diagnóstico y comorbilidades asociadas. La adherencia al tratamiento y la técnica inhalatoria también fueron evaluadas durante el seguimiento estrecho realizado por cada uno de los médicos tratantes.

\section{Modo de empleo de omalizumab}

La dosis y la frecuencia de administración (cada 2 o 4 semanas) dependerá del peso y del nivel de IgE total (Tabla 3 y 4). La dosis debe ajustarse sólo frente a cambios significativos en el peso. En dos de los pacientes reportados los niveles de IgE estaban por fuera de los límites establecidos por la tabla. A pesar de lo anterior los médicos tratantes decidieron utilizar el medicamento de igual forma debido a la gravedad de los pacientes y por nueva evidencia publicada en adultos que demuestra que Omalizumab es seguro y eficaz en pacientes con niveles de $\operatorname{IgE} /$ peso corporal por sobre los niveles aprobados en la tabla ${ }^{14}$. En este caso se administró la dosis

Tabla 3. Dosis de Omalizumab (mg/dosis) administrada por inyección subcutánea cada 4 semanas $^{13}$

\begin{tabular}{|c|c|c|c|c|c|c|c|c|c|c|}
\hline \multirow[b]{2}{*}{$\begin{array}{l}\text { IgE basal } \\
\text { UI/ml }\end{array}$} & \multicolumn{10}{|c|}{ Peso corporal (kg) } \\
\hline & $>20-25$ & $>25-30$ & $>30-40$ & $>40-50$ & $>50-60$ & $>60-70$ & $>70-80$ & $>80-90$ & $>90-125$ & $>125-150$ \\
\hline$>30-100$ & 75 & 75 & 75 & 150 & 150 & 150 & 150 & 150 & 300 & 300 \\
\hline$>100-200$ & 150 & 150 & 150 & 300 & 300 & 300 & 300 & 300 & 450 & 600 \\
\hline$>200-300$ & 150 & 150 & 225 & 300 & 300 & 450 & 450 & 450 & 600 & \\
\hline$>300-400$ & 225 & 225 & 300 & 450 & 450 & 450 & 600 & 600 & & \\
\hline$>400-500$ & 225 & 300 & 450 & 450 & 600 & 600 & \multirow{3}{*}{\multicolumn{4}{|c|}{$\begin{array}{c}\text { Administración cada } 2 \text { semanas } \\
\text { Véase la Tabla } 4\end{array}$}} \\
\hline$>500-600$ & 300 & 300 & 450 & 600 & 600 & & & & & \\
\hline$>600-700$ & 300 & & 450 & 600 & & & & & & \\
\hline
\end{tabular}

Tabla 4. Dosis de Omalizumab (mg/dosis) administrada por inyección subcutánea cada 2 semanas ${ }^{13}$

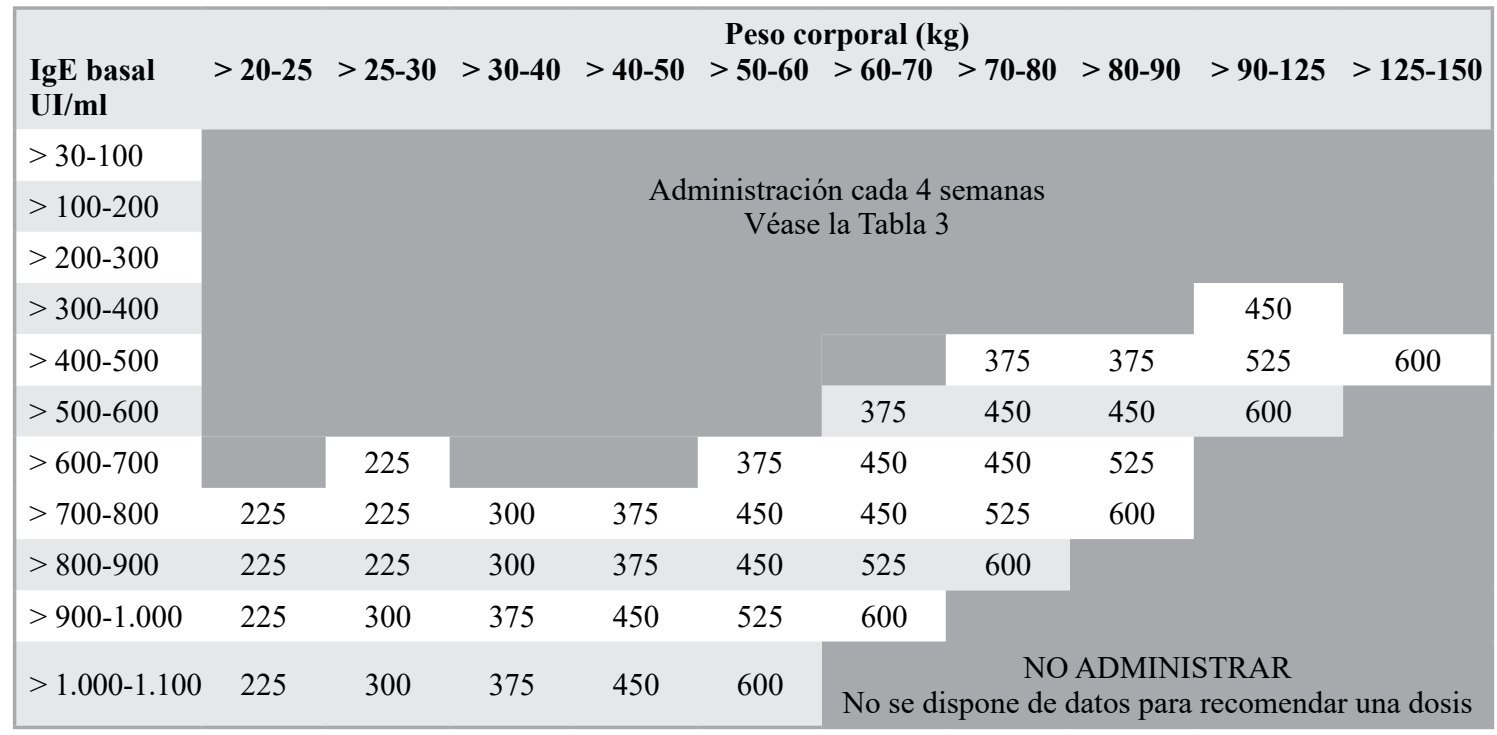


máxima de 600 mg cada 12 h, observándose una excelente respuesta clínica. Omalizumab se administra por vía subcutánea y está disponible en dos presentaciones: vial conteniendo $150 \mathrm{mg}$ en $5 \mathrm{ml}$ y jeringa precargada con $150 \mathrm{mg}$ en $1 \mathrm{ml}$. Se han comunicado algunos casos de reacción anafiláctica con el uso de Omalizumab, aunque es de muy rara ocurrencia, presentándose en menos del $0,1 \%$ de los pacientes tratados. Pese a lo anterior se recomienda que al momento de la administración haya un período de observación bajo supervisión médica de al menos $2 \mathrm{~h}$, especialmente durante las tres primeras dosis ${ }^{15}$. Una vez iniciado el tratamiento se recomienda evaluar la eficacia clínica a las 16 semanas, para lo cual se consideran varios índices como el número de exacerbaciones, número de consultas a Servicio de Urgencia, curas con corticoides sistémicos, hospitalizaciones, mejoría del nivel de control medido por cuestionario ACT o Asthma Control Questionnaire (ACQ) y/o mejoría de la calidad de vida ${ }^{16}$. La respuesta al tratamiento no debe controlarse con niveles de IgE total ya que estos resultan ser más altos que el nivel basal pretratamiento, debido a la formación de complejos Omalizumab-IgE que se eliminan más lentamente en comparación con la IgE libre. Si la respuesta clínica no es satisfactoria a las 16 semanas de iniciado el tratamiento se recomienda discontinuar, de lo contrario se debiera mantener. El tiempo total de tratamiento aún no está bien definido en la literatura, existiendo series con períodos de tratamiento que varían entre 4 a 6 años ${ }^{17,18}$. En nuestra serie los pacientes que discontinuaron la terapia por problemas de costo a los 4 y 6 meses tuvieron una recaída muy significativa de los síntomas. Esto no se observó en el paciente en que la terapia se suspendió luego de un año de uso. Este paciente ha tenido una buena evolución, sólo ha presentado hasta ahora dos crisis de asma que requirieron el uso de corticoides sistémicos por 3 días, sin necesidad de consultas a Servicio de Urgencia ni hospitalizaciones, manteniendo una buena tolerancia al ejercicio y calidad de vida.

\section{Eficacia y seguridad de omalizumab}

Desde el punto de vista clínico son múltiples las publicaciones que avalan la eficacia de Omalizumab, incluso luego de haber suspendido la terapia ${ }^{19}$. En un meta-análisis que incluyó a 3.261 pacientes con asma moderado a severo, que estaban en tratamiento con corticoides inhalados, se observó una reducción significativa de las exacerbaciones de asma, $26 \%$ en el grupo placebo versus $16 \%$ en el grupo tratado ${ }^{20}$.
También hubo una reducción significativa de las hospitalizaciones, $3 \%$ en el grupo placebo y $0,5 \%$ en el grupo tratado. Los pacientes que recibieron Omalizumab pudieron suspender completamente el tratamiento con corticoides inhalados en forma más frecuente que sus pares que recibieron placebo $(\mathrm{OR}=2,50 ; 95 \%$ IC: 2,00 a 3,13$)^{20}$. Resultados similares se observaron en otro meta-análisis que incluyó a 3.429 pacientes, en el que se objetivó una reducción significativa del riesgo de exacerbaciones en el grupo tratado en comparación con el grupo placebo $(\mathrm{RR}=0,57$; 95\% IC: $0,48-0,66 ; p=0,0001)^{21}$. Este efecto fue independiente de la duración del tratamiento, la edad y la severidad del asma. En este estudio también se observó que los pacientes tratados, a diferencia del grupo placebo, podían en forma más frecuente suspender por completo el uso de corticoides $(\mathrm{RR}=1,80 ; 95 \%$ IC: $1,42-2,28 ; \mathrm{p}=$ 0,00001). En estudios de la vida real Omalizumab ha demostrado reducir los síntomas de asma y el uso de terapia de rescate en más de un $50 \%$ con respecto al basal pre tratamiento y además reducir el uso de tratamiento con corticoides orales desde un $28,6 \%$ a un $14,2 \%{ }^{22}$. Otros beneficios reportados con el uso de Omalizumab son disminución de las consultas a Servicio de Urgencia, mejoría de la calidad de vida y reducción de los costos en salud, observándose un ahorro de 1.152 dólares (USA) por paciente respondedor a la terapia en 6 meses $^{23}$. Por último, el uso de este anticuerpo monoclonal en niños ha demostrado reducir las exacerbaciones estacionales que se producen año a año en los meses de otoño, producto de infecciones las virales al momento del inicio del año escolar $^{24}$. En este interesante estudio se observó que el promedio de exacerbaciones en el grupo placebo fue de $9 \%$ en otoño, $8,1 \%$ en primavera y $4,6 \%$ en verano $(p<0,001)$. En cambio, en el grupo tratado con Omalizumab no se observó esta variación estacional ya que el promedio de exacerbaciones en estos pacientes fue de $4,3 \%$ en otoño, $4,2 \%$ en primavera y $3,3 \%$ en verano. La diferencia entre el grupo placebo y Omalizumab fue significativa $(\mathrm{p}<0,001)$. Este efecto sería por restauración de los mecanismos de defensa antiviral ${ }^{9}$.

En nuestra serie pudimos observar que el tratamiento con Omalizumab produjo una reducción muy importante y en algunos casos desaparición por completo de las exacerbaciones de asma con disminución del uso de corticoides sistémicos, consultas al Servicio de Urgencia y hospitalizaciones. En tres casos en particular el uso de Omalizumab produjo una mejoría muy importante de los síntomas de asma diurnos, lo que 
permitió a los pacientes volver a realizar clases de educación física. La mejoría clínica asociada al uso de Omalizumab se acompañó además de una mejoría de la calidad de vida de los pacientes y de sus cuidadores.

Además de su eficacia Omalizumab tiene un buen perfil de seguridad. Los meta-análisis publicados al respecto demuestran que la frecuencia de efectos adversos serios es similar a placebo, $3,8 \%$ para Omalizumab y $5,3 \%$ para placebo $^{21}$. Los efectos adversos locales son más frecuentes con el uso de Omalizumab en comparación con placebo (19,9\% versus $13,2 \%)$. Los más frecuentemente reportados son equimosis, enrojecimiento, calor local o prurito en el sitio de inyección. En la mayoría de los casos los efectos adversos son de presentación leve y transitoria, tal como lo observamos en nuestros pacientes ${ }^{21,25}$.

\section{Comentario final}

La mayoría de los pacientes con asma en la edad pediátrica tienen un fenotipo alérgico con inflamación eosinofílica, por lo que responden adecuadamente al tratamiento con corticoides inhalados. Sin embargo, existe un subgrupo de pacientes severos, como los que presentamos en esta serie, en los que no se logra el control de la enfermedad a pesar de recibir tratamiento con dos o más controladores. Por su condición, estos pacientes tienen asociada una muy mala calidad de vida y su manejo implica importantes costos en salud. Es en estos pacientes en los que Omalizumab podría tener un efecto beneficioso. Sin embargo, para poder pensar en la utilización de este medicamento el paciente debe seguir un protocolo de estudio muy acucioso por parte del especialista, de manera de tener la certeza que su uso realmente se justifica. De esta manera se obtendrá un verdadero beneficio para el paciente y también para el sistema de salud, ya que a pesar de que el costo del tratamiento es elevado, éste es menor al gasto en salud asociado a la alta morbilidad de este tipo de pacientes.

\section{Bibliografía}

1.- WENZEL S. Severe asthma: from characteristics to phenotypes to endotypes. Clinical Exp Allergy 2012; 42: 650-8.

2.- FITZPATRICK A M, TEAGUE W G, MEYERS D A, PETERS S P, LI X, LI H, et al. Heterogeneity of severe asthma in childhood: confirmation by cluster analysis of children in the National Institutes of Health/National Heart, Lung, and Blood Institute Severe Asthma
Research Program. J Allergy Clin Immunol 2011; 127: 382-9.

3.- PELAIA G, VATRELLA A, MASELLI R. The potential of biologics for the treatment of asthma. Nat Rev Drug Discov 2012; 11: 958-72.

4.- GALLI S, TSAI M, PILIPONSKY A. The development of allergic inflammation. Nature 2008; 454 (7203): 445 54.

5.- WALSH G M. Severe eosinophilic asthma and mepolizumab. Lancet Respir Med 2016; 4: 528-9.

6.- HOLGATE S T. New strategies with anti-IgE in allergic diseases. World Allergy Organization Journal 2014; 7 : 17.

7.- LICARI A, MARSEGLIA A, CAIMMI S, CASTAGNOLI R, FOIADELLI T, BARBERI S, et al. Omalizumab in children. Paediatr Drugs 2014; 16: 491-502.

8.- ROTH M, ZHAO F, ZHONG J, LARDINOIS D, TAMM M. Serum IgE induced airway smooth muscle cell remodeling is independent of allergens and is prevented by omalizumab. PLoS One 2015 Sep 2; 10 (9): e0136549.

9.- TEACH S J, GILL M A, TOGIAS A, SORKNESS C A, ARBES S J JR, CALATRONI A. Preseasonal treatment with either omalizumab or an inhaled corticosteroid boost to prevent fall asthma exacerbations. J Allergy Clin Immunol 2015; 136: 1476-85.

10.- GINA 2016. Disponible en: www.ginasthma.com

11.- CHUNG K F, WENZEL S E, BROZEK J L, BUSH A, CASTRO M, STERK P J, et al. International ERS/ATS guidelines on definition, evaluation and treatment of severe asthma. Eur Respir J 2014; 43: 343-73.

12.- BUSH A, SAGLANI S. Management of severe asthma in children. Lancet 2010; 376: 814-25.

13.- HERRERA A M, ABARA S, ÁLVAREZ C, ASTUDILLO C, CORRALES R, CHALA E, et al. Consenso chileno SER-SOCHINEP para el manejo del asma severo en el niño. Rev Chil Enferm Respir 2016; 32: 100-12.

14.- KORNMANN O, WATZ H, FUHR R, KRUG N, ERPENBECK V J, KAISE G. Omalizumab in patients with allergic (IgE-mediated) asthma and IgE/bodyweight combinations above those in the initially approved dosing table. Pulm Pharmacol Ther 2014; 28: 149-53. doi: 10.1016/j.pupt.2014.03.003

15.- ZIELEN S, LIEB A, DE LA MOTTE S, WAGNER F, DE MONCHY J, FUHR R, et al. Omalizumab Protects against Allergen-Induced Bronchoconstriction in Allergic (Immunoglobulin E-Mediated). Asthma Int Arch Allergy Immunol 2013; 160: 102-10.

16.- KIM H, LEIGH R, BECKER A. Omalizumab: Practical considerations regarding the risk of anaphylaxis Allergy, Asthma and Clinical Immunology 2010; 6: 32.

17.- BOUSQUET J, SIERGIEJKO Z, SWIEBOCKA E, HUMBERT M, K. RABE F, SMITH N, et al. Persistency of response to omalizumab therapy in severe allergic (IgE-mediated) asthma. Allergy 2011; 66: 671-8.

18.- LAI T, WANG S, XU Z, ZHANG C, ZHAO Y, HU 
Y. Long-term efficacy and safety of omalizumab in patients with persistent uncontrolled allergic asthma: a systematic review and meta-analysis. Sci Rep 2015 Feb 3; 5: 8191 .

19.- NOPP A, JOHANSSON S G, ADÉDOYIN J, ANKERST J, PALMQVIST M, OMAN H. After 6 years with Xolair; a 3-year withdrawal follow-up. Allergy 2010; 65: 56-60.

20.- NORMANSELL R, WALKER S, MILAN S J, WALTERS E H, NAIR P. Omalizumab for asthma in adults and children. Cochrane Database Syst Rev 2014 Jan 13; (1): CD003559.

21.- RODRIGO G J, NEFFEN H, CASTRO-RODRÍGUEZ $\mathrm{J}$ A. Efficacy and safety of subcutaneous omalizumab vs placebo as add-on therapy to corticosteroids for children and adults with asthma: a systematic review. Chest 2011; 139: 28-35.
22.- BRAUNSTAHL G J, CHEN C W, MAYKUT R, GEORGIOU P, PEACHEY G, BRUCE J. The eXpeRience registry: the 'real-world' effectiveness of omalizumab in allergic asthma. Respir Med 2013; 107: 1141-51.

23.- HUMBERT $M$, BUSSE W, HANANIA N A, LOWE $P$ J, CANVIN J, ERPENBECK V J, et al. Omalizumab in asthma: an update on recent developments. J Allergy Clin Immunol Pract 2014; 2: 525-36.

24.- BUSSE W W, MORGAN W J, GERGEN P J, MITCHELL H E, GERN J E, LIU A H, et al. Randomized trial of omalizumab (anti-IgE) for asthma in inner-city children. N Engl J Med 2011; 364: 1005-15.

25.- HOLGATE S T, CHUCHALIN A G, HÉBERT J, LÖTVALL J, PERSSON G B, CHUNG K F, et al. Efficacy and safety of a recombinant anti-immunoglobulin E antibody (omalizumab) in severe allergic asthma. Clin Exp Allergy 2004; 34: 632-8.
Correspondencia a:

Dra. Ana María Herrera G.

Clínica Santa María, Santiago.

E mail: amherreragana@gmail.com 